\title{
Caretaker's Perceptions on Caretaker-Adolescent Communication on Sexual and Reproductive Health in Unguja-Zanzibar: Implication for Intervention
}

\author{
Saada A. Seif1, Thecla W. Kohi' ${ }^{1}$ Lilian T. Mselle² \\ ${ }^{1}$ Department of Nursing Management, School of Nursing, Muhimbili University of Health and Allied Sciences, \\ Dar es Salaam, Tanzania \\ ${ }^{2}$ Department of Clinical Nursing, School of Nursing, Muhimbili University of Health and Allied Sciences, \\ Dar es Salaam, Tanzania \\ Email: salis1969@hotmail.com,nakutz@yahoo.com
}

Received 5 March 2016; accepted 10 April 2016; published 13 April 2016

Copyright $@ 2016$ by authors and Scientific Research Publishing Inc.

This work is licensed under the Creative Commons Attribution International License (CC BY). http://creativecommons.org/licenses/by/4.0/

(c) (1) Open Access

\begin{abstract}
Caretaker-adolescent communication is an appealing source for influencing adolescents' knowledge, attitudes and behavior, because caretakers are an accessible and often willing source of information for their children. With the realization of the importance of caretakers in influencing adolescent behaviours, it becomes interesting to explore their perceptions about caretaker-adolescent communication on Sexual and Reproductive Health (SRH). An exploratory qualitative research design was conducted in all six districts of Unguja-Zanzibar. Twelve focus group discussions (FGDs) with caretakers who were purposively selected were conducted. Thematic analysis guided the interpretation of the data. The main themes that were identified are perceived the risk of adolescents; style, time and content of communication; barriers to parent-child communication on SRH; and helping our children pass through adolescence safely. The findings show that participants have acknowledged the apparent risky sexual behaviors of their adolescents, and that information on SRH should be offered to children before they reach puberty. Participants had the opinion that communication on SRH should be in the form of warning, threat and unidirectional. Topics such as teenage pregnancy, STIs and HIV, and drug and alcohol were proposed to be openly discussed. However, topics on condom and contraceptives use faced strong opposition. Lack of favorable time, caretakers' limited knowledge and skills, myth on SRH communication, and being uncomfortable were among the caretaker-adolescent communication barriers identified by care-
\end{abstract}


takers. The findings suggest that participants are keen to help their adolescents to avoid SRH problems, most importantly they emphasised on the need to empower caretakers by equipping them with knowledge of SRH, good communication skills and religious knowledge. Communal parenting on the other hand has been identified as the major weapon in shaping adolescents' behaviour. This information provides insight in designing family-based intervention aiming at increasing communication about SRH between caretakers and adolescents.

\section{Keywords}

Caretaker, Adolescent, Reproductive Health, Sexuality, IMB-Model, Qualitative Study

\section{Introduction}

Adolescents nowadays grow up in circumstances quite different from those of their parents, with greater access to formal education, increasing need for technological skills such as computer and internet literacy, different job opportunities, and more exposure to new ideas through media, telecommunications and other avenues [1]. However, this "widening world" often exposes adolescents to serious risks before they have adequate information, skills and experience to avoid or counteract them unless they are provided with support, information and access to resources [2]. Adolescents' participation in sexually risky behaviors is a source of concern to parents and adolescent health care providers worldwide [3]. This is mainly because sexually active adolescents engage in various practices that pose a risk to sexual health. For instance, early initiation of sexual activity prolongs the period of exposure to risk of contracting Sexually Transmitted Infections (STIs), Human Immunodeficiency syndrome virus (HIV), the potential for unplanned pregnancy, as well as greater likelihood of unprotected intercourse and multiple sexual partners [4] [5]. As in many parts of sub-Saharan Africa (SSA), sexual activity begins early in Tanzania. The social-demographic survey revealed that about $20 \%$ of the population had had sexual intercourse by the age of 18 years, and by age $15,11 \%$ of the girls and boys have had sex [6].

Caretakers (parents or guardians who live and take care of adolescent/adolescents) occupy a very important position to help socialise adolescents to healthy sexual behaviours, by providing accurate information about sex, health, personal development, transitioning to sexual life, and fostering responsible sexual decision-making [1] [7] [8]. Caretaker-adolescent communication is an appealing source for influencing adolescents' knowledge, attitudes and behavior, because caretakers are an accessible and often willing source of information for their children [9]. Therefore, through caretaker-adolescent communication on SRH matters, adolescents can be protected from SRH problems, including HIV infection [10] [11]. Most published studies on caretaker-adolescent communication on SRH have sought to find out whether or not parents communicate with their children about SRH matters [12]; while others have assessed the process of communication, and perceptions on quality and comfort of such communication [13] [14]. However, the vast majority of these studies have been conducted in North America, Europe and Australia [10]. In Sub-Sahara Africa (SSA), studies on caretaker-adolescent communication on SRH are increasing [1]. However, in most of these studies the participants have been young people, and most have adopted quantitative methodologies [15]-[18] that do not elicit rich, in-depth understanding of caretakers' perceptions on the topic.

In Tanzania, studies on caretaker-adolescent communication on SRH matters from the perspective of caretakers are very limited, and the few that are available, are primarily done in rural areas where social structures are different from social structures in urban areas [19] [20]. Therefore, there is a need for further exploration of the subject especially in Zanzibar which has its own unique cultural, traditional and religious differences [21]. This study focused on exploring caretakers' perceptions on the idea of communicating SRH matters with adolescents irrespective of whether they are discussing these matters with their children. Findings from this study will provide us with deeper understanding of caretakers' needs and attitudes towards SRH communication with adolescents, which are important factors for designing effective interventions with the purpose of increasing communication about SRH matters between caretakers/parents and adolescents.

\section{Theoretical Framework: Information-Motivation-Behavioural (IMB) Skills Model}

The IMB model identifies three core determinants of the initiation and maintenance of health behaviours: accu- 
rate information that can be readily translated into health behaviour performance; personal and social motivation to act on such information; and behavioral skills to confidently and effectively implement the health behaviour [22]. The IMB model explains that performing a health behaviour is a function of the extent to which someone is well-informed about the behaviour, motivated to perform the behaviour (e.g., has positive personal beliefs and attitudes towards the behaviour or outcome, perceiving vulnerability and social support to perform the behaviour), and has the requisite skills to execute the behaviour and confidence in their ability to do so across various situations.

\section{Methods}

\subsection{Design}

A descriptive exploratory study design was used to explore the perceptions of caretakers on caretaker-adolescent communication on SRH.

\subsection{Setting}

This study took place between September and November 2013 in the six districts of Unguja namely Urban, West, North A, North B, South and Central. According to the National Population and Housing Census of 2012, there were about 302,293 people aged between 35 and 60 years; among them, 147,470 were male and 154,823 were female. The social-demographic survey [7] revealed that about $20 \%$ of the population had had sexual intercourse by the age of 18 years and HIV statistics shows that about $265(1.2 \%)$ of people in Unguja have been infected, among them $114(43 \%)$ are young people aged between 15 and 24 years (however, it is not clear to us whether these were infected from birth, however they pose the risk of transmitting HIV).

\subsection{Informants}

Study participants were caretakers of adolescents aged between 15 - 19 years. Purposive sampling of maximum variation was used to select participants. This type of sampling is when the researcher relies on judgmental when it comes to selecting the study units for the purpose of focusing on particular characteristics of a population which will best enable to answer the research questions, and to gain greater insights into a phenomenon by looking at it from all angles which will help to identify common themes that are evident across the sample [23]. Participants were selected through Shehas (head of Shehia) following the given inclusion criteria. Shehia means the lowest administrative authority in the hierarchy of regional administration [24]. The inclusion criteria were: a caretaker who was from a religious institution, school teacher, health care worker, politician and a regular community member. The diverse selection of participants was intended to explore the varied perceptions within a community that could be influenced by the type of socialisation a person had. Ninety two participants were approached to participate in this study and eighty percent (80\%) which is equal to seventy-four caretakers agreed to take part and were recruited.

\subsection{Data Collection}

Twelve FGDs were conducted, six for males and six for females separately. All FGDs were held in schools when the students were away on weekend rest. The discussions were held in Kiswahili which is spoken by all participants and they were audio-recorded with participants' permission. Male focus group discussions were facilitated by trained same gender moderators and note takers, and female focus group discussions were facilitated by the principal investigator and trained female note takers. An average of eight participants took part in each discussion lasting for about one and half to two hours. The interview guide is found in Appendix 1.

\subsection{Data Analysis}

Thematic analysis [25] guided the interpretation of the data. Audio-recorded discussions were transcribed and manually coded. The analysis started by getting familiar with the data, generating initial codes and searching for categories and themes among codes. Themes were defined, refined, and logical connections established between them. The codes used to develop theme are found in Appendix 2. 


\subsection{Ethical Consideration}

The Muhimbili University of Health and Allied Sciences (MUHAS) Research Ethical Committee provided a go-ahead for the study to proceed. Permission to conduct the study was granted by the District Officers of six districts of Unguja, Zanzibar. Written consent was also obtained from caretakers after the purpose and methods of the study had been explained. It was made clear that any participant would be free to withdraw from the study any time and that information obtained from the study would be treated with strict confidentiality and will be used only for the intended research purposes. Participants were provided with the Principal Investigator's (PI) contacts so that they could report any cases of infringement upon their rights.

\section{Findings and Discussion}

The four themes that emerged from caretakers that were related to perceptions on caretaker-adolescent communication were: 1) Perceived risk of adolescents; 2) Style, time and content of communication; 3) Barriers to parent-child communication on SRH; and 4) Helping our children pass through adolescence safely. No differences in perception across age, sex and position in the society were observed.

\subsection{Characteristics of Participants}

Seventy-four caretakers participated in this study, among them 36 were males and 38 were females. Participants' age ranged between 35 and 60 years, and they described themselves as fishermen, farmers, government and private sectors employees and peasants. Social demographic characteristics of the participants are summarised in Table 1.

\section{Table 1. Demographic characteristics of participants.}

\begin{tabular}{|c|c|c|}
\hline Characteristics & Male & Female \\
\hline \multicolumn{3}{|l|}{ Age } \\
\hline $35-45$ years & 15 & 16 \\
\hline $46-60$ years & 21 & 22 \\
\hline \multicolumn{3}{|l|}{ Religious affiliation } \\
\hline Muslim & 36 & 38 \\
\hline \multicolumn{3}{|l|}{ Economic activities } \\
\hline Farmers & 7 & 6 \\
\hline Peasants & 5 & 9 \\
\hline Fishermen & 6 & 2 \\
\hline Employed & 18 & 21 \\
\hline \multicolumn{3}{|l|}{ Position in the society } \\
\hline Religious representatives & 6 & 6 \\
\hline Political representatives & 6 & 6 \\
\hline School teachers & 6 & 9 \\
\hline Healthcare workers & 6 & 6 \\
\hline Community members & 12 & 11 \\
\hline
\end{tabular}

\subsection{Perceived Risk of Adolescents}

Participants had concern about misuse of technology and information media that had put their adolescents at the risk of getting reproductive health problems like early sexual debut, teenage pregnancy, HIV and STIs, and unsafe abortion. And therefore they called for the need to provide SRH information to adolescents. They associated the uncontrolled use of technology and media with SRH risks. One of the participants complained. 
...Adolescents do not use information in the media to educate themselves, rather they use it to spoil their behaviour; for instance, they use social media and websites to send to each other pornographic images and videos, and finally unknowingly, they fall into risky habits... (50-year-old male, school teacher).

Another participant commented:

You might find an adolescent in possession of a mobile phone which contains only pornographic images and videos, and they also use the mobile phones to make contacts with their boyfriends... (35-year-old female, from community).

Disintegration of morals and values as a result of adolescents' lust for money, luxurious goods and materials; peer pressure; wearing indecent clothing; and drug and alcohol abuse, were also described as being responsible for adolescents' engagement into risky sexual behaviours. Participants acknowledged that poverty of parents/caretakers was to blame for such individuals' failure to provide for their young ones. One participant admitted:

...Most parents are poor, they cannot provide for their children's luxurious goods; and children now are more materialistic than we used to be. Now children could ask for money from anyone even if they didn't know them (58-year old male, from local government).

Another participant talked about peer pressure and craving for material things by adolescents:

I can say, things that spoil our children today is excessive leisure and being materialistic; when a girl sees a new fashionable item, she might get tempted to acquire it. Sometimes her friend might tell her, "Look at my new handbag? If you want one like this, I could show you how" (40-year-old female, from community).

The findings indicate that caretakers were aware of the sexual risks adolescents face. The inappropriate use of the internet and satellite television was believed to be associated with adolescent risk behaviours. Some studies have associated exposure to sexual contents through the media, with the development of risky sexual behaviour [26]. However, there is great potential for technology to improve the sexual and reproductive health of adolescents while reducing health risks through education, interventions, and provision of resources. For example, the development of websites in which users can find local STD/HIV testing centres; watch videos of celebrities talking about the importance of getting tested; and videos sharing which can allow for peer-to-peer health communications, can establish positive behavioural norms, provide role modelling, and effect change [27].

Looking at the general economic condition of the people in Unguja, where $36.8 \%$ live in severe poverty [28], and the increasing availability of luxurious and fashionable goods in the market, it is to be expected that caretakers would be unable to meet the luxurious demands of their children. Most adolescents therefore are believed to be falling into risky sexual behaviours just to be able to afford a few luxurious goods. For example, an adolescent may establish a sexual relationship with an older person provided she is assured of support-whether financial or material. If young people are made to meet their own basic material needs or/and are forced to provide the essential needs of their families, caretakers may lose both authority over these young people's decisions and actions (both sexual and non-sexual), and their ability to communicate to adolescents about their risky sexual behaviour [20].

\subsection{Style, Time and Contents of Communication}

\subsubsection{Style}

Some participants were of the opinions that for the adolescents to listen and obey, caretakers should shout, warn, promise to punish, and impose fear, as one participant said:

...I think the method of imposing fear is very helpful, you must be serious and speak out of rage, only then might the child be scared and get convinced that if he/she ever indulges in that act (sex), her/his parent would definitely punish him/her (58-year-old male, from community).

Another participant emphasised that SRH communication should be in the form of instructions and unidirectional rather than engage in a dialogue, as she said:

In these topics, there is nothing to discuss, what you need to do is issue warnings and instructions only, no dialogue (50-year-old female, from community).

Findings were consistent with what Wamoyi et al. (2010) observed, that parent-child communication often takes the form of warning, threat, and it is usually unidirectional and judgmental [19]. However in this study participants' suggestions went beyond shouting and creation of fear, to beating adolescents. One participant retorted: 
...Nowadays, they are telling us not to cane our children, but by just using words children will not listen to you; we have to cane them, that is the style of raising kids that was passed over to us by our parents. This new style of just using words is what is spoiling our children today (55-year-old male assistant medical officer).

Participants use these communication styles because they usually initiate this discussion late, when an adolescent has fallen into sexual risky behaviour, and thus SRH communication comes as a means of trying to deter that behaviour, as one participant said:

You know, we usually do not talk to our adolescents until we see them falling into risky behaviour, by that time we are like scolding them...but this is also a one way of giving education (40-year-old male from community).

The suggested communication styles create a barrier between caretakers and adolescents when communicating on SRH issues as was shown in our previous study [29]. Therefore, there is a need to impart appropriate communication skills to caretakers, to help them improve communication about SRH at the family level.

\subsubsection{Timing of Initiating SRH Communication-the Younger the Better}

Participants reported that SRH education/information should be given to children prior to puberty, because knowledge given at this age lasts for a long time. Further, globalisation is making children aware of issues related to SRH at a younger age, as one participant said:

In this period that we are in, you might find a mother as young as 12 years old; this is due to the effects of globalisation. Therefore, communication should start at the age of 8 years (50-year-old male primary school teacher).

Another participant added:

I think we should start giving them this education when they are 7 years old because this is the age during which a child starts to get aware about her/his sexual identity (40-year-old female from community).

Other participants had the opinion that SRH communication should start at even a younger age of 3 years, where simple topics related to morals and values should be included; and as the child grows, more complex topics like body physiological changes and risky habits, condom and contraceptives use could be taught:

We must be very careful, each stage of development is accompanied by its own body and behavioural changes, therefore we must know what to teach at each age, like we cannot teach a child of 8 or 9 years about how to prevent pregnancy or about use of condoms; this is not right (45-year-old male secondary school teacher).

These findings indicate that participants were aware of the significance of initiating SRH communication at an early age so as to have the desired impact on adolescents' SRH. However, they were much more concerned about providing age-appropriate information. These findings are encouraging and support the recommendation that it is crucial to initiate SRH discussions with young people before their sexual debut, to have the desired SRH impact [30].

\subsubsection{Contents of SRH Information}

Participants thought that topics of SRH such as teenage pregnancy, STIs, HIV, and drug and alcohol abuse, should be discussed openly:

...We should explain everything to both sexes openly, as we can now see that most children are using drugs and alcohol; therefore, we should teach them about the ill consequences of alcohol and drug use, and also about HIV/AIDS which can also be acquired through sharing contaminated needles (41-year-old female nurse).

Another participant added:

...We have to be open and tell them everything, not only that they would get pregnant if they initiate sexual activities early, but also to tell them all the expected consequences including contacting sexually transmitted diseases (37-year-old female, from the community).

Other topics mentioned were about preventive aspects like abstaining from sex, creating self-awareness, ways of avoiding temptations, and teaching of good norms. As one participant said:

“...We should teach them about self-awareness, because this is a very important attribute for any youth in order to protect self from temptations. And you must tell them about all the temptations that they might encounter at their age, and tell them about the associated risks like teenage pregnancy and STIs, and how they could protect themselves (45-year-old female secondary school teacher).

Very few mentioned the biological aspects of sexuality, including secondary sexual characteristics, the reproductive system, and fertile days. 
First, they should be taught about the reproductive system and how it functions, and also about physical developmental changes when such changes happen and what would follow those changes. They should also be taught about the significance of self-protection from the effects of those changes if they fail to protect themselves (36-year-old male secondary school teacher).

1) Communication about condoms and other contraceptives

Participants were generally against communication about condom use, with adolescents. Some warned that this kind of communication was against some religious beliefs and that discussing about such issues was totally immoral. Others suggested that condoms should not be allowed into the country because they are not effective in preventing diseases and pregnancies. A good number, (about $80 \%$ ) believed that if adolescents are taught about how condoms are used, this would be like giving them permission to practice sex. As one participant said:

...We should never mention these things (meaning condoms), because we are all Muslims here, and Allah forbids us from doing things that will harm us, because if you say children cannot abstain so they should use these things, you should know that Allah would not be pleased with this... (46-year-old female from madrasa).

Another participant added:

According to me, an adolescent should not be told about condom use, if you tell him/her to use a condom, it means you are giving him/her permission to practice sex. What you have to tell him/her is to abstain from sex, observe good norms and avoid all bad/risky behaviours (49-year-old male from community).

Participants claimed that contraceptives are not for adolescents; rather they are for married couples only. Family planning services in health facilities are said to be restricted to older women and not for adolescents, as one participant opined:

To say the truth, here in Zanzibar, family planning methods are allowed only for married women, but for these young adolescents, we do not have permission to give them yet (52-year-old female nurse).

All the findings that have been discussed above indicate that there is strong opposition in the use of condoms and other contraceptive methods especially to adolescents. This was expected, and it could be fuelled by the strong religious and cultural beliefs which proscribe premarital sexual intercourse. In Zanzibar, about $99 \%$ of the population is Muslim [31] and their culture is grounded in Islam of which law strongly prohibits premarital sexual activities [32]. Therefore, talking to adolescents about condoms and other contraceptives is perceived as encouraging them to practice sex, which is against their religious and cultural norms. Due to this, caution should be taken when introducing health programmes aiming at encouraging condom use especially to young ones. Effort like introducing education programmes to both caretakers and adolescents to encourage them to ignore cultural norms which seem to be unhelpful is needed.

\subsection{Barriers to Caretaker-Adolescent Communication on SRH}

Participants identified a number of barriers to communication about SRH with adolescents. The main obstacles mentioned include feeling embarrassed or uncomfortable by the participants' responses. One participant opined:

According to our cultural norms, to talk about these things (meaning SRH matters) to adolescents is shameful and it is taboo; it is like you are teaching them to do these things (meaning sex), therefore we opt not to tell them (42 year-old female from a health centre).

Another participant added:

We as parents are not used to communicating with our adolescents about sexuality, may be because it is not our culture to do so. My parents never talked to me about these things; therefore I also don't talk to my adolescents, that is to say we do not have that knowledge and skills of communicating with them (55 years, female school teacher).

These findings suggest that social and traditional norms of participants' culture do not offer a friendly environment where issues of SRH could be honestly and openly discussed. As in many parts of Africa, these findings are not surprising. A study done in Kenya showed that mothers do not talk to their adolescents because themselves had not received sex education from their own mothers [33]. The perception that talking about SRH encourages sex was also found in another study [34] which was conducted in Kenya and United State. Moreover, the knowledge barrier was also found in other studies which showed that parents generally felt that they lacked adequate information to share with their children [35] [36]. These findings signify that effort is needed to help these societies to re-examine their cultural norms and ignore those which seem to be unhelpful in confronting the new challenges, including the HIV. Caretakers need to be educated, motivated and provided with good 
communication skills to enable them engage in open communication about SRH with their adolescents.

\subsection{Helping Children Pass through Adolescence Safely}

Participants were very concerned about the reproductive health of their adolescents, and the idea of collaborative nurturing emerged. They emphasised the importance of bringing back the former upbringing style which involved collaboration of the whole society in disciplining the child. One participant said:

There is a need for parents today to be like former parents; we have to be united and allow our children to be reared by the society. We should not to get angry when our children get scolded by someone else for their wrong doing (56 years old, male caretaker).

Most importantly they emphasised empowering caretakers by equipping them with knowledge of SRH, good communication skills and religious knowledge. One participant said:

I insist, we need this knowledge, adolescents expect to get help from us but we cannot help them. Also we have to follow the teachings of our religion, all these problems will be overcome... (40 years, female school teacher).

The findings suggest that participants are keen to help their adolescents to avoid SRH problems. Communal parenting has been identified as the major weapon in shaping adolescents' behaviour. The significance of communal parenting was also highlighted in [20], in which participants claimed that traditional communal parenting make children upbringing and shaping their behaviour a lot easier.

\subsection{IMB-Model in Relation to This Study}

\subsubsection{Information Construct}

1) Contents and importance of SRH information

Participants were aware of the type of information on SRH that is recommended for adolescents, ranging from biological aspects, preventive aspects, to associated risk aspects. It was noted that participants were able to describe the associated risk aspects in detail, like how HIV is acquired and transmitted and how it can affect adolescents' life compared to other aspects like preventive aspects or biological aspects. However, significant differences in knowledge were observed between other community members, healthcare workers and school teachers. Both school teachers and health care workers were found to be considerably more knowledgeable on SRH matters. However, the participants' main concern was what to communicate, and at which stage of adolescence. They feared to give adolescents information that is not suitable for their age, like teaching about condom use to an 8-year-old child. However, participants still underscored the importance of communicating SRH information to their adolescents believing that it would definitely help them to refrain from risky sexual behaviours. This information is very important when planning for intervention programmes to increase caretakeradolescent communication on SRH.

\subsubsection{Motivation Construct}

1) Perceived risk: This study found that participants were aware of the sexual risk that their adolescents were in, and each caretaker was concerned about his/her own adolescents believing that they were also at the same risk as the rest of the population. This is encouraging because as per IMB model speculation, being able to perceive the risk gives a reason to initiate the behaviour.

2) Social norms: The findings of this study indicate that participants were aware of the significance of social support in meeting SRH needs of their adolescents. Participants were calling for collaboration and working as a team in their society to protect their adolescents from falling in risky sexual behaviours. The question about how best to communicate about condom use faced strong opposition from participants. This issue needs to be carefully handled when introducing any programme aimed at encouraging condom use to adolescents. For example, one may start by introducing condoms to caretakers themselves as a means of safe family planning. After this has been accepted, then one might encourage caretakers to communicate and introduce the same to their adolescents, and how to indulge in responsible sex should it become necessary.

3) Attitude: In general, participants showed positive attitude in communicating with adolescents about SRH. They took it to be a good thing to communicate with their adolescents about SRH, but this was only in selected age-appropriate topics. Moreover, Individual, social and cultural norms and religious belief of participants resulted in strong negative attitude towards communication about condom and contraceptive use to adolescents. This needs to be carefully addressed in any intervention programmes targeting caretakers to encourage them 
communicate SRH matters to their adolescents.

\subsubsection{Behavioural Skills}

1) Perceived self-efficacy: The findings of this study indicate that participants were not confident in communicating SRH information with adolescents, especially younger adolescents, as participants seem to emphasise that this education should be initiated in schools and madrasa (Islamic education institution). It was noted that participants would rather wait for something to trigger such communication like a case of teenage pregnancy, drug/alcohol use or abortion complications, for them to have the courage to initiate such communication.

2) Perceived skills: Participants acknowledged that they did not have enough skills to communicate with their adolescents about SRH information. This can be explained by the participants' response when they said, "with this issue there is no discussion"; they believed that caretakers must communicate with adolescents by giving instructions, accompanied by shouting, warning, threatening and even beating. These findings call for the needs to help caretakers to develop good communication skills so that they are able to positively influence their adolescents to refrain from risky sexual behaviours.

\section{Limitations}

Although the study findings might improve our understanding of caretakers' needs and attitude on SRH communication with adolescents, the research has had its limitations. Inclusion of health care workers and school teachers provided useful and interesting insights, however; their contributions and ideas may influence the response of other caretakers because they are considered as most knowledgeable on health related issues in the society and therefore may have resulted in a picture that reflects only those who were exposed to reproductive and child health education, leaving out the voices of those who were not exposed to that kind of education.

\section{Conclusion}

This study revealed that despite caretakers' awareness of the sexual risk on their adolescents face, recognising the importance of communicating with their adolescents and having positive attitude toward communicating with their adolescents on SRH, they are still not enthusiastic and remain uncomfortable when communicating about this matter. Lack of adequate knowledge about SRH, inadequate communication skills, inadequate parenting skills, myth attached to SRH communication, and conflicting societal norms were among the barriers of such communication. This observation has implications for professional social workers especially if practitioners are to design interventions at the family level that address the complex problems of unplanned pregnancy and STIs among adolescents.

\section{Acknowledgements}

We are very grateful to Sida Capacity Strengthening programme through the Muhimbili University of Health and Allied Sciences for funding this study. Immense thanks to all research assistants who were involved in data collection. And finally our gratitude goes to all study participants who shared their perceptions on caretakeradolescent communication on SRH with us.

\section{References}

[1] United Nation Population Fund (UNFPA) (2008) Generation of Change: Young People and Culture. Youth Supplement to UNFPA's State of the World Population Report, New York.

[2] World Health Organization (WHO) (2007) Helping Parents in Developing Countries Improve Adolescent's Health. World Health Organization, Geneva.

[3] Anarfi, K. (1992) Sexual Networking in Selected Communities in Ghana and the Sexual Behaviour of Ghanaian Female Migrants in Abidjan, Cote d'Ivoire. In: Dyson, T., Ed., Sexual Behaviour and Networking: Anthropological and Socio-Cultural Studies on the Transmission of HIV, Derouaux-Ordina Editions, Liege.

[4] United Nation (UN) (1990) Patterns of First Marriage: Timing and Prevalence. United Nations, Department of International Economic and Social Affairs, New York, 7-18.

[5] Bledsoe, C.H. and Cohen, B. (1993) Social Dynamics of Adolescent Fertility in Sub-Saharan Africa, Population Dynamics of Sub-Saharan Africa. National Academy Press, Washington DC, 45-56. 
[6] Population Council, Tanzania Commission for AIDS (TACAIDS), Zanzibar AIDS Commission (ZAC) and UNICEF Tanzania (2015) The Adolescent Experience In-Depth: Using Data to Identify and Reach the Most Vulnerable Young People, Tanzania 2009-2012. Dar es Salaam: Population Council, TACAIDS, ZAC and UNICEF Tanzania.

[7] Kumi-Kyereme, A., Awusabo-Asare, K., Biddlecom, A. and Tanle, A. (2007) Influence of Social Connectedness, Communication and Monitoring on Adolescent Sexual Activity in Ghana. African Journal of Reproductive Health, 11, 133-149. http://dx.doi.org/10.2307/25549736

[8] Zhang, L., Li, X., Shah, I., Baldwinc, W. and Stanton, B. (2007) Parent-Adolescent Sex Communication in China. The European Journal of Contraception and Reproductive Health Care, 12, 138-147.

http://dx.doi.org/10.1080/13625180701300293

[9] Lefkowitz, E.Z., Sigman. M. and Au, T.K. (2000) Helping Mothers Discuss Sexuality and AIDS with Adolescents. Child Development, 71, 1383-1394. http://dx.doi.org/10.1111/1467-8624.00234

[10] Bastien, S., Kajula, L.J. and Muhwezi, W.W. (2011) A Review of Studies of Parent-Child Communication about Sexuality and HIV/AIDS in Sub-Saharan Africa. Reproductive Health, 8, 25. http://dx.doi.org/10.1186/1742-4755-8-25

[11] Burgess, V., Dziegielewski, F.S. and Green, E.C. (2005) Improving Comfort about Sex Communication between Parents and Their Adolescents: Practice-Based Research within a Teen Sexuality Group. The Journal of Brief Treatment and Crisis Intervention, 5, 379-390. http://dx.doi.org/10.1093/brief-treatment/mhi023

[12] DiClemente, R.J., Wingood, G.M., Crosby, R., Cobb, B.K., Harrington, K. and Davies, S.L. (2001) Parent-Adolescent Communication and Sexual Risk Behaviours among African American Adolescent Females. Journal of Pediatrics, 139, 407-412. http://dx.doi.org/10.1067/mpd.2001.117075

[13] Feldman, S.S. and Rosenthal, D.A. (2000) The Effect of Communication Characteristics on Family Members' Perceptions of Parents as Sex Educators. Journal of Research on Adolescence, 10, 119-150. http://dx.doi.org/10.1207/SJRA1002_1

[14] Downing, J., Jones, L., Bates, G., Sumnall, H. and Bellis, M.A. (2011) A Systematic Review of Parent and FamilyBased Intervention Effectiveness on Sexual Outcomes in Young People. Health Education Research, 26, 808-833. http://dx.doi.org/10.1093/her/cyr019

[15] Adu-Mireku, S. (2003) Family Communication about HIV/AIDS and Sexual Behaviour among Senior Secondary School Students in Accra, Ghana. African Health Sciences, 3, 7-14.

[16] Amoran, O.E., Onadeko, M.O. and Adeniyi, J.D. (2005) Parental Influence on Adolescent Sexual Initiation Practices in Ibadan, Nigeria. International Quarterly of Community Health Education, 23, 73-81. http://dx.doi.org/10.2190/U8VJ-0UFE-HRYU-J48L

[17] Babalola, S., Tambashe, B.O. and Vondrasek, C. (2005) Parental Factors and Sexual Risk-Taking among Young People in Cote d'Ivoire. African Journal of Reproductive Health, 9, 49-65. http://dx.doi.org/10.2307/3583160

[18] Biddlecom, A., Awusabo-Asare, K. and Akinrinola, B. (2009) Role of Parents in Adolescent Sexual Activity and Contraceptive Use in Four African Countries. International Perspectives on Sexual and Reproductive Health, 35, 72-81. http://dx.doi.org/10.1363/3507209

[19] Wamoyi, J., Fenwick, A., Urassa, M., Zaba, B. and Stones, W. (2010) Parent-Child Communication about Sexual and Reproductive Health in Rural Tanzania: Implications for Young People's Sexual Health Interventions. Reproductive Health, 7, 6. http://dx.doi.org/10.1186/1742-4755-7-6

[20] Wamoyi, J., Fenwick, A., Urassa, M., Zaba, B. and Stones, W. (2011) Socio-Economic Change and Parent-Child Relationships: Implications for Parental Control and HIV Prevention among Young People in Rural North Western Tanzania. Culture, Health \& Sexuality: An International Journal for Research, Intervention and Care, 13, 615-628. http://dx.doi.org/10.1080/13691058.2011.562305

[21] Bakari, M.A. (2001) Democratisation Process in Zanzibar: A Retarded Transition. https://books.google.com/books?isbn=3928049712

[22] Fisher, J.D. and Fisher, W.A. (1992) Changing AIDS-Risk Behaviour. Psychological Bulletin, 111, 455-474. http://dx.doi.org/10.1037/0033-2909.111.3.455

[23] Laerd Dissertation (2012) Purposive Sampling. http://dissertation.laerd.com/purposive-sampling.php

[24] Bill Supplement to the Zanzibar Government Gazette Vol. No. CXXI I 6486 of 23th October (2014). http://www.zanzibarassembly.go.tz/bills/2014/A-Bill-of-Regional-Commission.pdf

[25] Braun, V. and Clarke, V. (2006) Using Thematic Analysis in Psychology. Qualitative Research in Psychology, 3, 77-101. http://dx.doi.org/10.1191/1478088706qp063oa

[26] Kachur, R., Mesnick, J., Liddon, N., Kapsimalis, C., Habel, M., David-Ferdon, C., Brown, K., Gloppen, K., Tevendale, H., Gelaude, D.J., Romero, L., Seitz, H., Heldman, A.B. and Schindelar, J. (2013) Adolescents, Technology and Reducing Risk for HIV, STDs and Pregnancy. Centers for Disease Control and Prevention, Atlanta. 
[27] Hoff, T., Rzepka, J., Cullins, V. and Friedman, A. (2010) Successes and Lessons Learned from the National GYT: Get Yourself Tested Campaign, a Public-Private Partnership Effort to Promote STD Testing among Sexually Active Youth. Panel Presented at the National Conference on Health Communication, Marketing, and Media, Atlanta. http://cdc.confex.com/cdc/nphic10/webprogram/Session11246.html

[28] Kessy, F. and Omar, M. (2014) Status and Progress of Human Development and Implications for Achieving Zanzibar Development Vision 2020. THDR 2014: Background Paper No. 10 ESRF Discussion Paper 55, Economic and Social Research Foundation.

[29] Seif, S.A. and Kohi, T.W. (2014) Caretaker-Adolescent Communication on Sexuality and Reproductive Health: My Perceptions Matter; A Qualitative Study on Adolescents' Perspectives in Unguja-Zanzibar. Health, 6, 2904-2917. http://dx.doi.org/10.4236/health.2014.621329

[30] Obasi, A.I., Cleophas, B., Ross, D.A., Chima, K.L., Mmassy, G., Gavyole, A., Plummer, M.L., Makokha, M., Mujaya, B., Todd, J., et al. (2006) Rationale and Design of the MEMA kwa Vijana Adolescent Sexual and Reproductive Health Intervention in Mwanza Region, Tanzania. AIDS Care, 18, 311-322.

[31] Wikipedia the free encyclopedia (2016) Zanzibar. https://en.wikipedia.org/wiki/Zanzibar

[32] Quran, Chapter Israa (17:32). http://tanzil.net/\#17:32

[33] Mbugua, N. (2007) Factors Inhibiting Educated Mothers in Kenya from Giving Meaningful Sex-Education to Their Daughters. Social Science \& Medicine, 64, 1079-1089. http://dx.doi.org/10.1016/j.socscimed.2006.10.008

[34] Poulsen, M.N., Miller, K.S., Lin, C., Fasula, A., Vandenhoudt, H., Wyckoff, S.C., Ochura, J., Obong'o, C.O. and Forehand, R. (2010) Factors Associated with Parent-Child Communication about HIV/AIDS in the United States and Kenya: A Cross-Cultural Comparison. AIDS and Behavior, 14, 1083-1094. http://dx.doi.org/10.1007/s10461-009-9612-4

[35] Guilamo-Ramos, V., Soletti, A.B., Burnette, D., Sharma, S., Leavitt, S. and McCarthy, K. (2012) Parent-Adolescent Communication about Sex in Rural India: U.S.-India Collaboration to Prevent Adolescent HIV. Qualitative Health Research, 22, 788-800. http://dx.doi.org/10.1177/1049732311431943

[36] Mahajan, P. and Sharma, N. (2005) Parents' Attitude towards Imparting Sex Education to Their Adolescent Girls. Anthropologist, 7, 197-199. 


\section{Appendix 1}

\section{An Interview Guide for Focus Group Discussion}

1) Do you think it is important for caretakers to communicate with adolescents about sexual and reproductive health? Explain why.

Probe:

- What are the sexual risky behaviors displayed by their adolescents?

- Which ages do most adolescents initiate sexual activities?

2) Do you think who is the right person to provide SRH information to adolescent? Explain why. Probe:

- Which is the best age of adolescent to initiate SRH communication?

- Which sex of caretaker should communicate SRH information to a boy and to a girl?

- Which topics of SRH should be discussed to adolescents?

- Should condom and contraceptive information be included in the discussion?

3) Do you think caretakers communicate with their adolescents about SRH information? Probe:

- What are the barriers that caretakers face when need to communicate with their adolescents about SRH?

- What do you think are the barriers that adolescents face when trying to reach caretakers to get SRH information? 


\section{Appendix 2}

\section{Caretakers' Perceptions on Caretaker-Adolescent Communication on SRH}

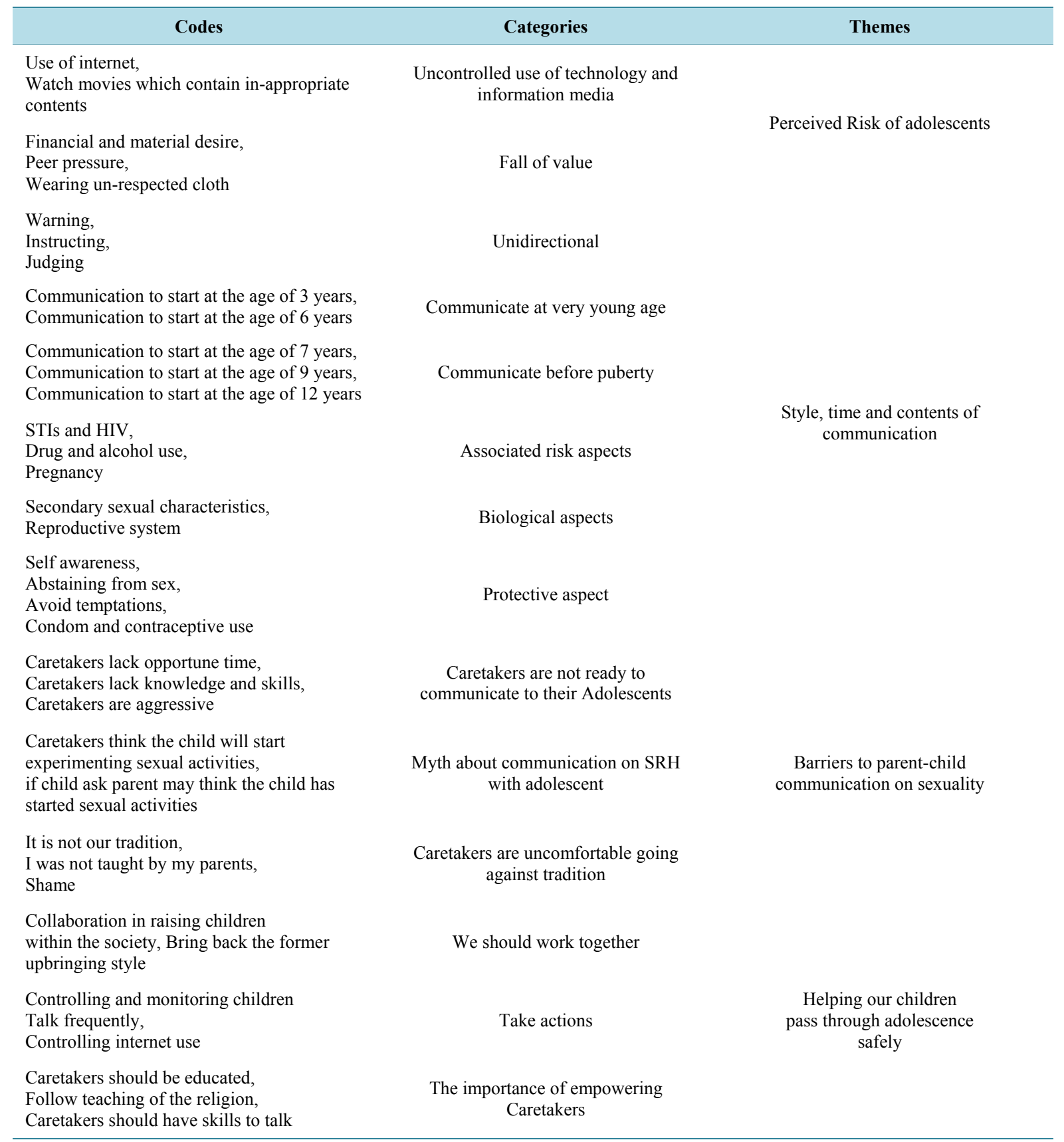

